\title{
Metazoários parasitos de Thyrsitops lepidopoides (Osteichthyes: Gempylidae) do litoral do Estado do Rio de Janeiro, Brasil
}

\author{
Metazoan parasites of Thyrsitops lepidopoides (Osteichthyes: \\ Gempylidae) from the coastal zone of the state of Rio de Janeiro, Brazil
}

\author{
Sarah Abdel Melek Domingues ${ }^{1}$ \\ Dimitri Ramos Alves ${ }^{2}$ \\ 1 Graduação em Ciências Biológicas, Ênfase em Biotecnologia, Centro Universitário de Volta Redonda - UniFOA. \\ 2 Docente do Curso de Ciências Biológicas do Centro Universitário de Volta Redonda - UniFOA. dimitri.alves@foa.org.br
}

\section{Resumo}

Entre outubro de 2011 e junho de 2012, foram coletados 55 espécimes da lanceta, Thyrsitops lepidopoides (Cuvier, 1832) (Osteichthyes: Gempylidae) provenientes do litoral do Estado do Rio de Janeiro (21-23ㅇ, 41-45ㅇ), sendo necropsiados para estudo de sua comunidade de metazoários parasitos. Dezenove espécies de metazoários parasitos foram coletadas: seis espécies de digenéticos, três de monogenéticos, uma de cestóide, três de acantocéfalos, quatro de nematóides, uma de copépode e uma de isópode. Thyrsitops lepidopoides é um novo registro de hospedeiro para dezessete espécies de metazoários parasitos. Todos os espécimes de T. lepidopoides estavam parasitados por, pelo menos, uma espécie de metazoário, totalizando 2430 espécimes de parasitos, com média de 44,2 \pm 33,1 por hospedeiro. A maioria dos espécimes foi de digenéticos (63,8\%), seguidos de nematóides $(17,9 \%)$ e de acantocéfalos (16,2\%). Lecithochirium microstomum Chandler, 1935 (Digenea: Hemiuridae) correspondeu à maioria dos espécimes de parasitos coletados $(\mathrm{n}=$ 1.490), sendo a espécie dominante, com os maiores valores de prevalência, abundância média, intensidade e intensidade média. Os parasitos apresentaram típico padrão de distribuição superdisperso (ID = 24,746; d $=41,352$ ). A abundância e a prevalência de quatro e dois parasitos, respectivamente, assim como o total de espécimes coletados $\left(r_{s}=0,671 ; P=<0,001\right)$ e a riqueza parasitária $\left(3,7 \pm 1,2 ; r_{s}=0,423 ; P=<0,001\right)$ apresentaram correlação com o comprimento total do hospedeiro.

\section{Palavras-chave}

Thyrsitops lepidopoides; Gempylidae; metazoários parasitos.

\begin{abstract}
From October 2011 and June 2012, 55 specimens of white snake mackerel, Thyrsitops lepidopoides (Cuvier, 1832) (Osteichthyes: Gempylidae) collected from coast of the State of Rio de Janeiro (21 - 23 $\left.\mathrm{S}, 41-45^{\circ} \mathrm{W}\right)$, were necropsied to study their communities of metazoan parasites. Nineteen metazoan parasites species were collected: six digeneans, three monogeneans, one cestode, three acanthocephalans, four nematodes, one copepod and one isopod. Thyrsitops lepidopoides is a new host record for seventeen parasite species. All fishes were parasitized by at least one metazoan species. Two thousand four hundred and thirty specimens, with an average of $44.2 \pm 33.1$ per host, were collected. The majority of the parasites specimens collected were digeneans (63.8\%), followed by the nematodes (17.9\%) and acanthocephalans (16.2\%). Lecithochirium microstomum Chandler, 1935 (Digenea: Hemiuridae) constituted the majority of specimens collected ( $n$ = 1490), was the dominant species, with highest prevalence, abundance, intensity and mean intensity. The parasites species showed typical over-dispersed pattern of distribution (ID $=24.746 ; d=41.352$ ). The abundance and the prevalence of four and two parasite species, respectively, the total parasites collected $\left(r_{s}=0.671 ; P=<0.001\right)$ and parasite species richness $\left(3.7 \pm 1.2 ; r_{s}=0.423 P=<0.001\right)$ showed correlation with host total length.
\end{abstract}

\section{Keywords}

Thyrsitops lepidopoides; Gempylidae, metazoan parasites.

\section{Como você deve citar?}

DOMINGUES, Sarah Abdel Melek; ALVES, Dimitri Ramos. Metazoários parasitos de Thyrsitops lepidopoides (Osteichthyes: Gempylidae) do litoral do Estado do Rio de Janeiro, Brasil. Cadernos UniFOA, Volta Redonda, n. 27, p. 95-103, abr. 2015. 


\section{INTRODUÇÃO}

Thyrsitops lepidopoides (Cuvier, 1832) (Perciformes: Trichiuroidea: Gempylidae) é um peixe ósseo marinho de pequeno porte, atingindo $40 \mathrm{~cm}$ de comprimento padrão. No sudeste brasileiro é conhecido como cavalinha do norte ou lanceta. Alimenta-se de pequenos peixes e crustáceos eufausiáceos e calanóides. É um gempilídeo mesobentopelágico que ocorre do Estado do Rio de Janeiro até cerca de $44^{\circ} \mathrm{S}$ de latitude, na Argentina e, também, no Chile (NAKAMURA e PARIN, 1993; LOWE-MCCONNEL, 1999; FIGUEIREDO e MENEZES, 2000; FIGUEIREDO et al., 2002).

Os estudos sobre os metazoários parasitos da lanceta são escassos. Dentre esses, podemos destacar os realizados por Vicente e Santos (1972), com o registro do nematóide Procamallanus macaensis, Vicente e Santos (1972) (Camallanidae), e por Vicente e Santos (1973), com o anisaquídeo Raphidascaris sp. (Anisakidae), P. macaensis e o digenético Lecithochirium microstomum Chandler, 1935 (Hemiuridae). Tais parasitos também foram registrados nos estudos realizados por Vicente et al. (1985) e Luque et al. (2011), com os nematóides, e por Kohn et al. (2007) com o digenético. Em relação aos ectoparasitos, podemos destacar o estudo realizado por Sartor (1986), com o registro do isópode Lironeca redmani Leach, 1818, parasitando T. lepidopoides. Recentemente, Luque et al. (2013) incluíram o registro supracitado na lista de crustáceos parasitos de peixes do Brasil.

O presente trabalho teve como objetivo desenvolver uma análise ecológica da comunidade de metazoários parasitos da lanceta, $T$. lepidopoides, proveniente do litoral do Estado do Rio de Janeiro, realizando uma análise da infracomunidade e comunidade componente.

\section{MATERIAL E MÉTODOS}

Entre outubro de 2011 e junho de 2012, foram necropsiados 55 espécimes de T. lepidopoides, provenientes do litoral do Estado do Rio de Janeiro (21 - $\left.23^{\circ} \mathrm{S}, 41-45^{\circ} \mathrm{O}\right)$. Os peixes foram adquiridos de pescadores artesanais. Uma vez obtidos, os mesmos foram acondicionados em caixas de isopor contendo gelo, para assegurar boas condições da coleta dos parasitos e protegê-los durante o transporte até o Laboratório de Zoologia do Centro Universitário de Volta Redonda (UniFOA), onde foram realizadas as necropsias. A identificação e a obtenção dos dados biométrico (comprimento total) e biológico (sexo) dos espécimes de T. lepidopoides foram feitas de acordo com Nakamura e Parin (1993) e Figueiredo e Menezes (2000). A coleta, registro e processamento dos parasitos foram realizados de acordo com os procedimentos indicados por Amato et al. (1991) e Eiras et al. (2000). A análise estatística foi aplicada somente para as espécies com prevalência maior que 10\% (BUSH et al., 1990). O cálculo da frequência de dominância e da dominância relativa (número de espécimes de uma espécie/número total de espécimes de todas as espécies de cada infracomunidade) foi feito seguindo a metodologia de Rohde et al. (1995). O quociente entre a variância e a abundância média (índice de dispersão) foi calculado para cada espécie de parasito com o intuito de determinar seu padrão de distribuição, sendo sua significância testada com o estatístico $d$ (LUDWIG e REYNOLDS, 1988). O coeficiente de correlação por postos de Spearman, $r$, foi usado para determinar possíveis correlações entre o comprimento total do hospedeiro e a abundância de infecção/ infestação. $O$ coeficiente de correlação de Pearson, $r$, foi usado para determinar a possível correlação entre o comprimento total do hospedeiro e a prevalência da infecção/infestação parasitária, com prévia transformação angular dos dados de prevalência (ZAR, 1996). As amostras dos hospedeiros foram separadas em quatro intervalos de classe com amplitude de $3,5 \mathrm{~cm}$. A influência do sexo na abundância e na prevalência das infecções parasitárias foi testada pela aproximação normal $Z c$ do teste $U$ de Mann-Whitney e pelo teste Qui-quadrado $\left(\chi^{2}\right)$, respectivamente. A terminologia ecológica usada é a recomendada por Bush et al. (1997). Todos os valores que correspondem à média de alguma variável são acompanhados do respectivo desvio padrão. O nível de significância estatística adotado foi $P<0,05$. 


\section{RESULTADOS}

\subsection{Componentes da comunidade parasitária}

Os espécimes de $T$. lepidopoides mediram $30,8 \pm 3,5(22,3-36) \mathrm{cm}$ de comprimento total. Os espécimes machos $(n=07)$ mediram $25,7 \pm 1,5 \mathrm{~cm}$ e as espécimes fêmeas $(n=48)$ mediram $31,6 \pm 3$ $\mathrm{cm}$ de comprimento total. 0 comprimento total dos espécimes machos e fêmeas apresentou diferença significativa $(t=-4,955 ; P<0,001)$.

Todos os espécimes de T. lepidopoides estavam parasitados por, pelo menos, uma espécie de metazoário. Thyrsitops lepidopoides é um novo registro de hospedeiro para dezessete espécies de metazoários parasitos. Um total de 2.430 espécimes de parasitos pertencentes a dezenove espécies foi coletado, com abundância média de 44,2 $\pm 33,1$. 0 digenético Lecithochirium microstomum foi o mais prevalente e abundante (Tabela 1). Os digenéticos, nematóides e os acantocéfalos corresponderam a $63,8 \%, 17,9 \%$ e 16,2\% do total de parasitos coletados, respectivamente. Os componentes da comunidade parasitária do T. lepidopoides apresentaram o típico padrão de distribuição superdisperso (Tabela 2). Lecithochirium microstomum apresentou a maior frequência de dominância e o maior valor de dominância relativa média (Tabela 3). A abundância e a prevalência parasitária apresentaram correlação com o comprimento total do hospedeiro em quatro e duas espécies de parasitos, respectivamente (Tabela 4). A abundância média $\left(r_{s}=0,671 ; P=<0,001\right)$ e a riqueza parasitária $\left(3,7 \pm 1,2 ; r_{s}=0,423 ; P=<0,001\right)$ apresentaram relação com o comprimento total do hospedeiro. Em relação ao sexo do hospedeiro, apenas a abundância do digenético $L$. microstomum $(U=3,158 ; P=0,001)$ e o total de parasitos $(U=$ $3,145 ; P=0,001)$ apresentaram correlação. 
Tabela 1 - Prevalência, amplitude da intensidade, intensidade média e abundância média dos metazoários parasitos de Thyrsitops lepidopoides do litoral do Estado do Rio de Janeiro, Brasil.

\begin{tabular}{|c|c|c|c|c|}
\hline Parasitos & Prevalência (\%) & $\begin{array}{l}\text { Amplitude da } \\
\text { intensidade }\end{array}$ & $\begin{array}{l}\text { Intensidade } \\
\text { média }\end{array}$ & $\begin{array}{l}\text { Abundância } \\
\text { média }\end{array}$ \\
\hline \multicolumn{5}{|l|}{ Digenea } \\
\hline Aponurus laguncula ${ }^{+}$ & 27,3 & $1-11$ & $2,8 \pm 2,7$ & $0,76 \pm 1,87$ \\
\hline Didimozoídeo imaturo $^{+}$ & 3,6 & 2 & 2 & $0,07 \pm 0,37$ \\
\hline 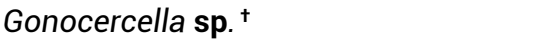 & 1,8 & --- & 1 & $0,01 \pm 0,13$ \\
\hline Lecithochirium microstomum & 96,4 & $1-100$ & $28,1 \pm 23,5$ & $27,1 \pm 23,7$ \\
\hline Paramphistomiformes gen. sp. ${ }^{\dagger}$ & 1,8 & --- & 1 & $0,02 \pm 0,13$ \\
\hline Rhipidocotyle sp. ${ }^{+}$ & 12,7 & $1-4$ & $1,71 \pm 1,45$ & $0,22 \pm 0,71$ \\
\hline \multicolumn{5}{|l|}{ Monogenea } \\
\hline Encotyllabe souzalimae ${ }^{\dagger}$ & 1,8 & --- & 1 & $0,02 \pm 0,13$ \\
\hline Octoplectanocotyla travassosi ${ }^{\dagger}$ & 21,8 & $1-8$ & $2,75 \pm 2,2$ & $0,6 \pm 1,51$ \\
\hline Pseudempleurosoma guanabarensis ${ }^{\dagger}$ & 7,3 & $1-4$ & $2 \pm 1,4$ & $0,14 \pm 0,62$ \\
\hline \multicolumn{5}{|l|}{ Cestoidea } \\
\hline 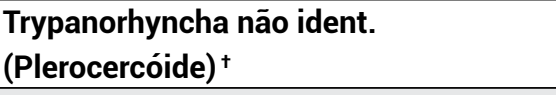 & 1,81 & --- & 1 & $0,02 \pm 0,13$ \\
\hline \multicolumn{5}{|l|}{ Acanthocephala } \\
\hline Corynosoma australe (Cistacanto) ${ }^{\dagger}$ & 3,6 & --- & 1 & $0,03 \pm 0,2$ \\
\hline 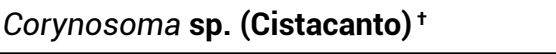 & 89,1 & $1-36$ & $7,9 \pm 7,4$ & $7,11 \pm 7,41$ \\
\hline Golvanorhynchus golvani ${ }^{+}$ & 1,8 & --- & 1 & $0,02 \pm 0,13$ \\
\hline \multicolumn{5}{|l|}{ Nematoda } \\
\hline Anisakis sp. (Larva) ${ }^{\dagger}$ & 7,3 & $1-4$ & $1,75 \pm 1,5$ & $0,13 \pm 0,6$ \\
\hline Contracaecum sp. (Larva) ${ }^{\dagger}$ & 67,3 & $1-109$ & $11,2 \pm 18,9$ & $7,5 \pm 16,3$ \\
\hline Pseudoterranova sp. (Larva) ${ }^{\dagger}$ & 5,4 & $1-2$ & $2,75 \pm 1,7$ & $0,1 \pm 0,32$ \\
\hline \multicolumn{5}{|l|}{ Nematoda } \\
\hline Raphidascaris sp. (Larva) & 7,3 & $1-5$ & $2,75 \pm 1,7$ & $0,2 \pm 0,82$ \\
\hline \multicolumn{5}{|l|}{ Copepoda } \\
\hline 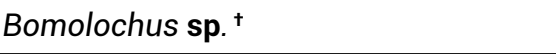 & 12,7 & --- & 1 & $0,12 \pm 0,33$ \\
\hline \multicolumn{5}{|l|}{ Isopoda } \\
\hline Gnathia sp. ${ }^{+}$ & 1,8 & --- & 1 & $0,02 \pm 0,13$ \\
\hline
\end{tabular}

(†) Novo registro de hospedeiro

Tabela 2 - Índice de dispersão (ID) e do estatístico d dos metazoários parasitos de Thyrsitops lepidopoides do litoral do Estado do Rio de Janeiro, Brasil. 


\begin{tabular}{ccc}
\hline Parasitos & Índice de dispersão (ID) & d \\
\hline Aponurus laguncula & 4,605 & $11,957 *$ \\
\hline Lecithochirium microstomum & 20,707 & $36,946 *$ \\
\hline Rhipidocotyle sp. & 2,323 & $5,495^{*}$ \\
\hline Octoplectanocotyla travassosi & 3,801 & $9,916^{*}$ \\
\hline Corynosoma sp. (Cistacanto) & 7,724 & $\mathbf{1 8 , 5 3 8 *}$ \\
\hline Contracaecum sp. (Larva) & 35,354 & $51,447 *$ \\
\hline Bomolochus sp. & 0,887 & 0,556 \\
\hline Total & $\mathbf{2 4 , 7 4 6}$ & $\mathbf{4 1 , 3 5 2 *}$
\end{tabular}

(*) Valores significativos.

Tabela 3 - Frequência de dominância, frequência de dominância compartilhada e dominância relativa média dos componentes das infracomunidades de metazoários parasitos de Thyrsitops lepidopoides do litoral do Estado do Rio de Janeiro, Brasil.

\begin{tabular}{lccc}
\hline \multicolumn{1}{c}{ Parasitos } & $\begin{array}{c}\text { Freqüência de } \\
\text { dominância }\end{array}$ & $\begin{array}{c}\text { Frequiência de dominância } \\
\text { compartilhada }\end{array}$ & $\begin{array}{c}\text { Dominância relativa } \\
\text { média }\end{array}$ \\
\hline Aponurus laguncula & 0 & 0 & $0,015 \pm 0,033$ \\
\hline Lecithochirium microstomum & 43 & 0 & $0,592 \pm 0,252$ \\
\hline Rhipidocotyle sp. & 0 & 0 & $0,003 \pm 0,009$ \\
\hline Octoplectanocotyla travassosi & 1 & 0 & $0,023 \pm 0,069$ \\
\hline Corynosoma sp. (Cistacanto) & 7 & 0 & $0,191 \pm 0,177$ \\
\hline Contracaecum sp. (Larva) & 4 & 0 & $0,145 \pm 0,188$ \\
\hline Bomolochus sp. & 0 & 0 & $0,005 \pm 0,020$ \\
\hline
\end{tabular}

Tabela 4 - Valores do coeficiente de correlação por postos de Spearman (rs) e do coeficiente de correlação de Pearson (r) para avaliar o relacionamento entre o comprimento total do Thyrsitops lepidopoides e a abundância e a prevalência dos componentes de sua comunidade parasitária.

\begin{tabular}{ccccc}
\hline Parasitos & rs & P & r & P \\
\hline Aponurus laguncula & $0,274^{*}$ & 0,042 & 0,924 & 0,076 \\
\hline Lecithochirium microstomum & $0,592^{*}$ & $<0,001$ & 0,356 & 0,643 \\
\hline Rhipidocotyle sp. & $0,284^{*}$ & 0,035 & $0,975^{*}$ & 0,025 \\
\hline Octoplectanocotyla travassosi & $-0,113$ & 0,407 & $-0,988^{*}$ & 0,012 \\
\hline Corynosoma sp. (Cistacanto) & 0,158 & 0,249 & $-0,328$ & 0,671 \\
\hline Contracaecum sp. (Larva) & $0,414 *$ & $<0,001$ & 0,451 & 0,549 \\
\hline Bomolochus sp. & 0,550 & 0,689 & 0,676 & 0,324 \\
\hline
\end{tabular}

(*) Valores significativos. $(P)$ nível de significância

\section{DISCUSSÃo}

No presente estudo pode-se observar a dominância de endoparasitos na comunidade parasitária de $T$. lepidopoides. 0 digenético $L$. microstomum foi a espécie dominante seguido pelo acantocéfalo Corynosoma sp. (estágio larval - cistacanto) e pelo nematóide Contracaecum sp. (estágio larval). A dominância de endoparasitos é um padrão que tem sido observado com frequência em comunidades 
parasitárias de peixes marinhos do Estado do Rio de Janeiro (MARQUES e ALVES, 2011; GONÇALVES e ALVES, 2012). No caso de T. lepidopoides, pode-se atribuir tal fato ao hábito alimentar carnívoro especializado em pequenos peixes, crustáceos eufausiáceos e moluscos (NAKAMURA e PARIN, 1993; FIGUEIREDO e MENEZES, 2000), potenciais hospedeiros intermediários/paratênicos de digenéticos, acantocéfalos e nematóides (MARCOGLIESE, 2002; LUQUE e POULIN, 2004; POULIN et al., 2008). Lecithochirium microstomum é um hemiurídeo eurixeno que possui uma ampla distribuição geográfica, sendo registrado por diversos autores, em hospedeiros nos Oceanos Atlântico, Indico e no Pacífico (MANTER, 1961; GIBSON e BRAY, 1979, 1986; BRAY, 1990, 1991; BRAY et al., 1993; GIBSON, 2002). No Brasil, esse hemiurídeo foi registrado parasitando peixes marinhos pertencentes às famílias Carangidae, Gempylidae, Gerreidae, Sciaenidae, Serranidae, Scombridae e Trichiuridae (KOHN et al., 2007). Além da dominância do digénético supracitado, duas outras espécies de larvas de parasitos (Corynosoma sp. e Contracaecum sp.) apresentaram elevados valores de prevalência e abundância. As formas larvais constituíram $42 \%$ dos táxons (oito espécies) da comunidade de metazoários parasitos de $T$. lepidopoides. A presença desses parasitos sugere que, mesmo sendo um peixe carnívoro, $T$. lepidopoides ocupe um nível intermediário na cadeia alimentar (ANKENBRANDT, 1985; LUQUE e POULIN, 2004).

Embora T. lepidopoides seja um peixe abundante na zona costeira da região sul e sudeste do Brasil (FIGUEIREDO et al., 2002), os estudos sobre a fauna parasitária desse peixe são escassos. Tal fato é reforçado pelos resultados obtidos no presente trabalho, onde foi realizado o registro, pela primeira vez, de dezessete espécies de metazoários parasitos em $T$. lepidopoides. Das três espécies de parasitos registrados em $T$. lepidopoides no Brasil, apenas o nematóide $P$. macaensis não foi registrado no presente estudo. Em relação aos aspectos quantitativos, os dados referentes às espécies Raphidascaris sp. e Lecithochirium microstomum (VICENTE e SANTOS, 1973) não são suficientes para possibilitar uma análise comparativa com os resultados obtidos. Vicente e Santos (1973) registraram o nematóide Porrocaecum sp. (Anisakidae) no hospedeiro conhecido como lanceta. Embora esse também seja um dos nomes vulgares utilizados para $T$. lepidopoides, tal registro não foi considerado pelo presente estudo.

O caráter agregado ou superdisperso da distribuição dos parasitos na amostra de T. lepidopoides é considerado um aspecto típico do parasitismo em função da amplitude das dimensões dos nichos e da heterogeneidade das populações de hospedeiros. A maioria dos estudos relacionados às comunidades de metazoários parasitos de peixes marinhos tem apresentado o padrão superdisperso (LUQUE et al., 1996; SILVA et al., 2000 a; MARQUES e ALVES, 2011; GONÇALVES e ALVES, 2012). 0 valor de dispersão não significativo determinado para Bomolochus sp. pode ser atribuído à baixa prevalência e intensidade desse tipo de copépode em $T$. lepidopoides.

Em T. lepidopoides, cinco espécies de parasitos apresentaram correlação entre o comprimento total do hospedeiro e a prevalência e/ou abundância parasitárias. Dentre esses, quatro espécies (Aponurus laguncula Looss, 1907, L. microstomum, Rhipidocotyle sp. e Contracaecum sp.) apresentaram correlação positiva e apenas o monogenético Octoplectanocotyla travassosi (CARVALHO e LUQUE, 2012) apresentou correlação negativa. A influência do comprimento do hospedeiro na composição qualitativa e quantitativa das comunidades parasitárias de peixes marinhos da costa brasileira tem sido discutida por diversos autores (ALVES e LUQUE, 2001; LUQUE et al., 2004; ALVES e LUQUE, 2006; MARQUES e ALVES, 2011). Entretanto, o que se observa nesses estudos é uma considerável heterogeneidade de resultados.

A comunidade parasitária de $T$. lepidopoides apresentou semelhança na sua composição em relação ao trichiurideo Trichiurus lepturus Linnaeus, 1758 (Perciformes: Trichiuroidea: Trichiuridae) estudado por Silva et al. (2000a, 2000b) e Carvalho e Luque (2011). Em ambos estudos, o digenético L. microstomum foi o parasito mais prevalente e abundante. Tal fato pode ser atribuído à biologia, ecologia, distribuição geográfica e filogenia desses hospedeiros (NAKAMURA, PARIN, 1993). Segundo 
esses autores(1993), T. lepidopoides apresenta distribuição vertical mesobentopelágica e T. lepturus bentopelágica, ocorrendo dessa forma sobreposição dos nichos alimentares.

\section{AGRADECIMENTO}

Agradecemos ao Centro Universitário de Volta Redonda (UniFOA) pelo apoio financeiro.

\section{REFERÊNCIAS}

ALVES, D.R.; LUQUE, J.L. Community ecology of the metazoan parasites of the White Croaker Micropogonias furnieri (Osteichthyes: Sciaenidae), from the coastal zone of the State of Rio de Janeiro, Brazil. Memórias do Instituto Oswaldo Cruz, v 96, n. 2, p. 145-153, 2001.

ALVES, D.R.; LUQUE, J.L. Ecologia das comunidades de metazoários parasitos de cinco espécies de escombrídeos (Perciformes: Scombridae) do litoral do Estado do Rio de Janeiro, Brasil. Revista Brasileira de Parasitologia Veterinária, v. 15, n. 4, p. 167-181, 2006.

AMATO, J.F.R.; BOEGER, W.A.; AMATO, S.B. Protocolos para Laboratório - Coleta e Processamento de Parasitos de Pescado. Imprensa Universitária, Universidade Federal Rural do Rio de Janeiro, 81p., 1991.

ANKENBRANDT, L. Food habits of bait-caught skipjack tuna, Katsuwonus pelamis, from the Southwestern Atlantic Ocean. Fishery Bulletin, v. 83, n. 3, p. $379-393,1985$.

BRAY, R.A. Hemiuridae (Digenea) from marine fishes of the southern Indian Ocean: Dinurinae, Elytrophallinae, Glomericirrinae and Plerurinae. Systematic Parasitology, v. 17, p. 183-217, 1990.

BRAY, R.A. Hemiuridae (Digenea) from marine fishes of the southern Indian Ocean: Genus Lecithochirium Lühe, 1901 (Lecithochiriinae). Systematic Parasitology, v. 18, p. 193-219, 1991.

BRAY, R.A.; CRIBB, T.H.; BARKER, S.C. The Hemiuroidea (Digenea) of pomacentrid fishes (Perciformes) from Heron Island, Queensland, Australia. Systematic Parasitology, v. 24, p. 159-184, 1993.

BUSH, A.O.; AHO J.M.; KENNEDY, C.R. Ecological versus phylogenetic determinants of helminth parasite community richness. Evolutionary Ecology, v. 4, n. 1. p. 1-20, 1990.

BUSH, A.O.; LAFFERTY, K.D.; LOTZ, J.M.; SHOSTAK, A.W. Parasitology meets ecology on its own terms: Margolis et al. revisited. Journal of Parasitology, v. 83, n. 4, p. 575- 583, 1997.

CARVALHO, A.R.; LUQUE, J.L. Seasonal variation in metazoan parasites of Trichiurus lepturus (Perciformes: Trichiuridae) of Rio de Janeiro, Brazil. Brazilian Journal of Biology, v. 71, n. 3, p. 771-782, 2011.

EIRAS, J.C.; TAKEMOTO, R.M.; PAVANELLI, G.C. Métodos de estudo e técnicas laboratoriais em parasitologia de peixes. Ed. Universidade Estadual de Maringá, Maringá. 171p., 2000.

FIGUEIREDO, J.L.; MENEZES, N.A. Manual de Peixes Marinhos do Sudeste do Brasil VI. Teleostei (5), Museu de Zoologia, Universidade de São Paulo, São Paulo, 116p., 2000. 
FIGUEIREDO, J.L.; SANTOS, L.P.; YAMAGUTI, N.; BERNARDES, R.A.; ROSSI-WONGTSCHOWSKI, C.L.D.B. Peixes da Zona Econômica Exclusiva da Região Sudeste-Sul do Brasil. São Paulo: Editora da Universidade de São Paulo, 248p., 2002.

GIBSON, D.I. Family Hemiuridae Looss, 1899. In: Gibson, D.I., Jones, A. \& Bray, R.A. (Eds) Keys to the Trematoda. Volume 1. Wallingford: CAB International. 521p., 2002.

GIBSON, D.I.; BRAY, R.A. The Hemiuridae (Digenea) of fishes from the north-east Atlantic. Bulletin of the British Museum Natural History, Zoology Series, v. 51, p. 1-125, 1986.

GIBSON, D.I.; BRAY, R.A. The Hemiuroidea: terminology, systematics and evolution. Bulletin of the British Museum (Natural History), Zoology Series, v. 36, p. 35-146, 1979.

GONÇALVES, P.H.S.; ALVES, D.R. Ecologia da comunidade de metazoários parasitos do xixarro, Trachurus lathami Nichols, 1920 (Osteichthyes: Carangidae) do litoral do Estado do Rio de Janeiro, Brasil. Cadernos UniFOA, v. 20, p. 105-113, 2012.

KOHN, A.; FERNANDES, B.M.M.; COHEN, S.C. South American trematodes parasites of fishes. 318p., 2007.

LOWE-MCCONNEL, R.H. Estudos Ecológicos de Comunidades de Peixes Tropicais. São Paulo: Editora da Universidade de São Paulo, 534p., 1999.

LUDWIG, J.A.; REYNOLDS, J.F. Statistical Ecology: a primer on methods and computing. New York: Wiley-Interscience Publications, 337p., 1988.

LUQUE, J.L.; POULIN, R. Use of fish as intermediate hosts by helminth parasites: A comparative analysis. Acta Parasitologica, v. 49, n. 4, p. 353-361, 2004.

LUQUE, J.L.; AMATO, J.F.R.; TAKEMOTO, R.M. Comparative analysis of the communities of metazoan parasites of Orthopristis ruber and Haemulon steindachneri (Osteichthyes: Haemulidae) from southeastern Brazilian littoral: I structure and influence of size and Sex of hosts. Revista Brasileira de Biologia, v. 56, n. 2, p. 279-292, 1996.

LUQUE, J.L.; MOUILLOT, D.; POULIN, R. Parasite biodiversity and its determinants in coastal marine teleost fishes of Brazil. Parasitology, v.128, p. 671 - 682, 2004.

LUQUE, J.L.; AGUIAR, J.C.; VIEIRA, F.M.; GIBSON, D.I.; SANTOS, C.P.. Checklist of Nematoda associated with the fishes of Brazil. Zootaxa, v. 3082, p. 1-88, 2011.

LUQUE, J.L.; VIEIRA, F.M.; TAKEMOTO, R.M.; PAVANELLI, G.C.; EIRAS, J.C. Checklist of Crustacea parasitizing fishes from Brazil. Check List, v. 9, n. 6, p. 1449-1470, 2013.

MANTER, H.W. Studies on digenetic trematodes of fishes of Fiji. I. Families Haplosplanchnidae, Bivesiculidae, and Hemiuridae. Proceedings of the Helminthological Society of Washington, v. 28, p. 67-74, 1961.

MARCOGLIESE, D.J. Food webs and the transmission of parasites to marine fish. Parasitology, v. 124, p. 83-89, 2002. 
MARQUES, L.C.; ALVES, D.R. Ecologia da comunidade de metazoários parasitos do dourado, Coryphaena hippurus Linnaeus, 1758, (Osteichthyes: Coryphaenidae) do litoral do Estado do Rio de Janeiro, Brasil. Cadernos UniFOA, v. 16, p. 111-122, 2011.

NAKAMURA, I.; PARIN, N.V. FAO species catalogue. Vol. 15. Snake mackerels and cutlassfishes of the world (Families Gempylidae and Trichiuridae). An annotated and illustrated catalogue of the snake mackerels, snoeks, escolars, gemfishes, sackfishes, domine, oilfish, cutlassfishes, scabbardfishes, hairtails, and frostfishes known to date. FAO Fisheries Synopis, v. 125, n. 15, p.1-136, 1993.

POULIN, R.; LUQUE, J.L.; GUILLAUMON, F.; MOUILLOT, D. Species abundance distributions and numerical dominance in gastrointestinal helminth communities of fish hosts. Journal of Helminthology, v. 82, p. 193-202, 2008.

ROHDE, K.; HAYWARD, C.; HEAP, M. Aspects of the ecology of metazoan ectoparasites of marine fishes. International Journal for Parasitology, v. 25, n. 8, p. 945-970, 1995.

SARTOR, S.M. Incidência de isópodes parasitas (Cymothoidae) em peixes da plataforma continental Brasileira. Boletin do Instituto Oceanográfico, v. 34, n. 1, p. 1-12, 1986.

SILVA, L.O.; ALVES, D.R.; LUQUE, J.L.; PARAGUASSÚ, A.R. Ecologia da comunidade de metazoários parasitos do Peixe-Espada Trichiurus lepturus Linnaeus (Osteichthyes, Trichiuridae) do litoral do Estado do Rio de Janeiro, Brasil. Revista Brasileira de Zoociências, v. 2, n. 2, p. 115-133, 2000a.

SILVA, L.O.; ALVES, D.R.; LUQUE, J.L. Metazoários parasitos do Peixe-Espada Trichiurus lepturus Linnaeus (Osteichthyes, Trichiuridae) do litoral do Estado do Rio de Janeiro, Brasil. Parasitología Al Día, v. 24, n. 3-4, p. 97-101, 2000b.

VICENTE, J.J.; SANTOS, E. Sobre um novo nematódeo camalanideo parasito de peixe marinho (Nematoda: Camallanoidea). Atas da Sociedade de Biologia do Rio de Janeiro, v. 15, n. 3, p. 145-147, 1972.

VICENTE, J.J.; RODRIGUES, H.O.; GOMES, D.C. Nematóides do Brasil. $1^{\text {a }}$ parte: Nematóides de peixes. Atas da Sociedade de Biologia do Rio de Janeiro, v. 25, p. 1-79, 1985.

VICENTE, J.J.; SANTOS, E. Alguns helmintos de peixes do litoral norte Fluminense - I. Memórias do Instituto Oswaldo Cruz, v. 71, n. 1-2, p. 95-113, 1973.

ZAR, J.H. Biostatistical Analysis. Upper Saddle River: Prentice-Hall, 662p., 1996. 